Abstract P70 Table 1 Baseline and compliance data of bariatric and sleep patients. Data presented as median (inter-quartile range) or n(\%). BMI: body mass index; ODI: overnight desaturation index; ESS: Epworth Sleepiness Score.

\begin{tabular}{lccc}
\hline & Bariatric (n= 49) & Sleep (n= 50) & p-value \\
\hline Age & $49(42-52)$ & $56(44-63)$ & 0.012 \\
Gender (F:M) & $29: 20$ & $20: 30$ & 0.056 \\
BMI (kg/m $)$ & $49.9(46.4-55.9)$ & $42.3(33-48.5)$ & $<0.001$ \\
$\begin{array}{l}\text { Baseline ODI (Average } \\
\text { dips/hour) }\end{array}$ & $31.1(22.3-43.7)$ & $41.0(21.5-61.6)$ & 0.280 \\
$\begin{array}{l}\text { Baseline ESS } \\
\text { Proportion of patients }\end{array}$ & $12(8-15)$ & $11(7.5-15)$ & 0.504 \\
$\begin{array}{l}\text { with } \geq 75 \% \text { of nights } \\
\text { CPAP use }\end{array}$ & $21(42.9 \%)$ & $25(50.0 \%)$ & 0.476 \\
\hline $\begin{array}{l}\text { Proportion of patients } \\
\text { with mean use of } \geq \mathbf{4}\end{array}$ & $20(40.9 \%)$ & & \\
hours per night & & & 0.087 \\
\hline
\end{tabular}

period. CPAP compliance at 4 weeks was compared between the two groups.

Results 409 patients were screened through the bariatric pathway during the study period. $49(12.0 \%)$ patients were diagnosed with OSA and were commenced on CPAP. Baseline characteristics of the bariatric and sleep clinic groups are shown in Table 1. There was no significant difference between gender, baseline overnight desaturation indices and baseline Epworth Sleepiness Scale scores between the two groups.

21 (42.9\%) patients in the bariatric group used their CPAP for $\geq 75 \%$ of nights within the 28 -day period compared with 25 $(50.0 \%)$ patients in the sleep clinic group; $p=0.48$. There was a trend to significance for proportion of patients who used CPAP for mean $\geq 4$ hours per night (20 (40.9\%)) patients in the bariatric group vs $29(58.0 \%)$ patients in the sleep clinic group; $p=0.087)$. Conclusion Our study suggests that the number of patients who are compliant with CPAP at mean use of $\geq 4$ hours per night may be lower in those with confirmed OSA found on routine screening within a bariatric pathway than in patients referred to a sleep clinic, but our finding was limited by the small sample size. Future study to investigate this trend and its underlying causes could improve the success of intervention in the bariatric patients.

\section{P71 CAN POSTURAL OSA BE IDENTIFIED FROM OXIMETRY ALONE?}

${ }^{1}$ A Johar, ${ }^{1} \mathrm{CD}$ Turnbull, ${ }^{2}$ JR Stradling. 'Oxford Centre for Respiratory Medicine, Oxford University Hospitals NHS Foundation Trust, UK; ${ }^{2}$ NIHR Oxford Biomedical Research Centre, University of Oxford, UK

\subsection{6/thoraxjnl-2016-209333.214}

Introduction Positional treatments have a long history of usage in obstructive sleep apnoea (OSA). Recent developments of more sophisticated therapies reported good response in patients with an AHI of $<60$ and predominately supine OSA (arbitrarily defined as total AHI: non-supine AHI $\geq 1.5) .{ }^{1,2}$ We hypothesised that patients with mild to moderate OSA usually have periods both with and without OSA, and that the OSA periods will be mainly due to supine OSA. Such patients might therefore be trialled on positional therapies without specific assessment of posture.

Methods Patients included had OSA and underwent an in-hospital respiratory sleep study between May- July 2016. Sleep studies included video review for identification of supine sleep. The oxygen desaturation index $>4 \%$ (ODI) was recorded in supine and non-supine positions. The ratio of the total to non-supine ODI was calculated.

Results 40 patients' sleep studies were suitable for analysis. 7 patients were excluded: 3 due to comorbidities (hypoventilation/ CSA), 3 due to lack of supine sleep ( $<30$ minutes) and one due to having minimal OSA. Patients had a mean age of 53.8 years (SD 12.7) and mean BMI of $34.9 \mathrm{~kg} / \mathrm{m}^{2}$ (SD 9.0). The median supine ODI was significantly higher than the median total ODI (supine ODI 54.3/h, IQR 47.9; total ODI 28.6/h, IQR 34.4; $\mathrm{p}<0.001)$. There was a significant relationship between the ratio of total ODI to non-supine ODI versus the total ODI (Spearman's rho $=0.37, \mathrm{p}=0.02$; Figure 1 ).

Discussion Our data suggests that patients with lower ODIs are more likely to have supine predominant OSA. At ODI values over 40 it is very unlikely that there is a posturally dependant component to a patient's OSA. However, the reverse is not the case; although many patients with low ODIs do have a postural component, many do not. It is therefore necessary to objectively assess the degree of supine predominant OSA in future trials of positional therapies, but patients with ODIs over 40 could be excluded at the outset. 


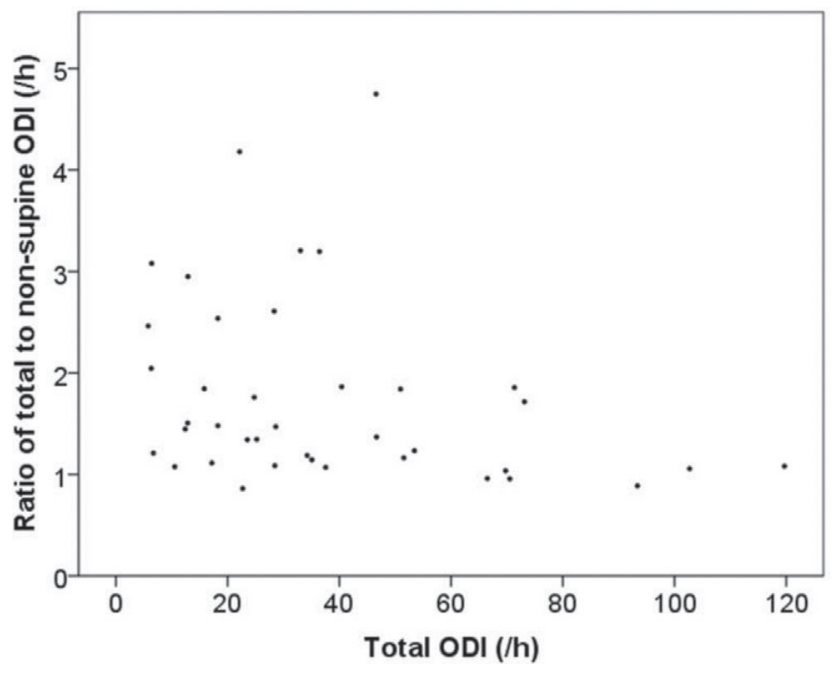

Abstract P71 Figure 1 The relationship between the total ODI and the ration of total to non-supine ODI

\section{REFERENCES}

1 Levendowski DJ, et al. Assessment of a neck-based treatment and monitoring device for positional obstructive sleep apnea. J Clin Sleep Med 2014;10(8):86371.

2 Levendowski DJ, et al. Capability of a neck worn device to measure sleep/wake, airway position, and differentiate benign snoring from obstructive sleep apnea. J Clin Monit Comput 2015;29:53-64.

\section{P72 BASELINE DATA FROM THE ROSA TRIAL: A RANDOMISED CONTROLLED TRIAL OF THE EFFECT OF CPAP ON DIABETIC MACULAR OEDEMA IN PEOPLE WITH CONCURRENT OBSTRUCTIVE SLEEP APNOEA}

SD West, J Hughes, B Prudon. NUTH, Newcastle, UK

\subsection{6/thoraxjnl-2016-209333.215}

The ROSA trial (Retinopathy and Obstructive Sleep Apnoea) is a multi-centre randomised controlled trial conducted in the United Kingdom. The hypothesis is that CPAP (continuous positive airway pressure) will improve visual acuity in people with diabetic macular oedema and concurrent OSA, due to improvements in intermittent hypoxia, blood pressure and catecholamine surges. An uncontrolled study showed visual acuity improved equivalent to one line on the logMAR chart in those people who used CPAP regularly at six months (Mason RH et al. Respiration 2012). We present baseline data from a larger randomised controlled trial.

Methods Patients of Eye Hospitals across the UK with diabetic macular oedema and type 2 diabetes were offered home sleep studies to diagnose OSA. These were posted to them with instructions by the coordinating centre and returned by post after a single night's recording. Those patients found to have severe OSA (ODI > 20 or AHI > 30), along with visual impairment due to diabetic macular oedema were randomised to usual ophthalmic care (control) or usual ophthalmic care plus CPAP for one year. Anyone with respiratory failure, excessive daytime sleepiness requiring urgent treatment or cataract precluding ophthalmic assessment was excluded. Follow up occurred at three, six and twelve months and included measures of sleepiness, health related quality of life, visual acuity, optical coherence tomography and retinal photography.

\section{Abstract P72 Table 1}

\begin{tabular}{llll}
\hline & $\begin{array}{l}\text { CPAP } \\
\mathrm{N}=64\end{array}$ & $\begin{array}{l}\text { Control } \\
\mathrm{N}=66\end{array}$ & P value \\
\hline Age (years) & $65.3(10.5)$ & $63.7(9.0)$ & n.s. \\
$\%$ male & 67 & 79 & \\
Body mass index $\left(\mathrm{kg} / \mathrm{m}^{2}\right)$ & $34.9(8.7)$ & $35.1(6.3)$ & n.s. \\
Neck circumference $(\mathrm{cm})$ & $43.2(4.1)$ & $44.9(4.0)$ & 0.017 \\
Duration of diabetes (years) & $16.3(8.7)$ & $15.2(9.5)$ & n.s. \\
HbA1c (mmol/l) & $67.0(17.5)$ & $66.7(23.1)$ & n.s. \\
Oxygen desaturation index/hr & $37.2(18.3)$ & $35.9(15.1)$ & n.s. \\
\hline
\end{tabular}

Results There have been 130 patients randomised from 23 UK centres; 64 to CPAP, 66 to control. The groups are well matched at baseline (Table).

Conclusions This novel study demonstrates that it is feasible to conduct a multicentre randomised controlled trial with UK Eye Hospitals and their local Sleep service, all coordinated by a single centre (Newcastle). The UK NHS National Institute for Health Research has facilitated this research via the Local Clinical Research Network at each centre. Minimisation criteria for randomisation has enabled the two groups to be well matched at baseline, essential for this type of study. The results of this trial will determine whether CPAP could form a novel treatment for diabetic macular oedema and and visual impairment in people with concurrent obstructive sleep apnoea; these results are eagerly awaited when follow up is completed in 2017.

\section{P73 ACUTE NON INVASIVE VENTILATION (NIV)-RELATED NASAL BRIDGE PRESSURE ULCERATION: EFFECT OF A PROACTIVE PREVENTION APPROACH}

${ }^{1} \mathrm{G}$ Stygall, ${ }^{1} \mathrm{~K}$ Morley, ${ }^{1} \mathrm{~L}$ Pickup, ${ }^{1} \mathrm{~A}$ Oakes, ${ }^{1} \mathrm{P}$ Antoine-Pitterson, ${ }^{2} \mathrm{~B}$ Chakraborty, ${ }^{1} \mathrm{R}$ Mukherjee. ${ }^{1}$ Heart of England NHS Foundation Trust, Birmingham, UK; ${ }^{2}$ School of Mathematics, University of Birmingham, Birmingham, UK

\subsection{6/thoraxjnl-2016-209333.216}

Introduction NIV applied via full face masks is increasingly becoming the standard of care in the treatment of acute hypercapnic respiratory failure. Most guidelines suggest good skin care: strategies include regular pressure relief, use of masks with softer cushions/pressure-avoidance masks and application of pressure-relieving dressing to the skin to redistribute pressure and reduce friction. We set out to examine the effect of a systematic proactive prevention approach to prevent Grade 2 Pressure ulcers in a Ward Based Physiotherapy-led acute NIV service in a general hospital serving a population of about 400000 . This included (a) prophylactic protective dressing and (b) reactive change to a pressure-avoidance mask for identified Grade 1 pressure sore.

Methods Data was collected from 01/05/2014 and 31/08/2015 which included an 8-month period before (period1) and an 8 month period after (period2) introduction of the proactive prevention approach. Five main sets of data were collected; the NIV mask used (model and size), whether the mask was changed, the total number of days using NIV, pressure ulcer grading and the outcome of the NIV admission. A pressure ulcer was defined as Grade 2 or above.

Results Grade 2 Pressure ulcer rates showed a trend in reduction by over 50\% following the change in practice (but fell short of statistical significance: chi-squared test $\mathrm{p}$-value $=0.3$ ). In period1 